\title{
Assessment of an ultrasound-guided technique for catheterization of the caudal thoracic paravertebral space in dog cadavers
}

\author{
Roger Medina Serra ${ }^{{ }^{*}}$, Carolina Palacios Jimenez ${ }^{1}$, Paolo Monticelli ${ }^{1}$, Mark Plested ${ }^{1}$ and Jaime Viscasillas ${ }^{2}$ \\ ${ }^{1}$ Department of Clinical Sciences and Services, The Royal Veterinary College, Hatfield, UK \\ ${ }^{2}$ Departament de Medicina y Cirugia Animal, Facultad de Veterinaria, Instituto de Ciencias Biomedicas, \\ Universidad CEU Cardenal Herrera, Valencia, Spain
}

\begin{abstract}
Background: The caudal thoracic paravertebral (CTPV) block is a regional anesthesia technique currently used in human medicine to provide analgesia in abdominal surgical procedures.

Aim: The objectives of this study are to describe an ultrasound-guided technique to place catheters in CTPV space in canine cadavers and evaluate the distribution of a 50:50 contrast-dye solution administered through them.

Methods: Eight thawed adult beagle cadavers $(9.2 \pm 2.0 \mathrm{~kg}$ body total weight $)$ were used. Thirteen catheters were placed. In the first phase, a volume of $0.3 \mathrm{ml} \mathrm{kg}$-1 of the contrast-dye was administered in all cases. After the injections, computed tomography $(\mathrm{CT})$ scans were carried out to assess the distribution of the contrast-dye. In the second phase, an extra $0.2 \mathrm{ml} \mathrm{kg}^{-1}$ of the contrast-dye was administered through eight catheters, followed by a second CT scan. Two cadavers were dissected to assess the distribution of the contrast-dye. The injection site varied between T8-9 and T12-13.

Results: The evaluation of the CT scans showed contrast-dye within the paravertebral space in $92 \%(12 / 13)$ of the injections. The distribution pattern observed after the injections performed within the TPV space was linear and intercostal in all cases. The median (range) linear spread of the contrast was 7 (5-10) spinal nerves and involved 3 (2-8) intercostal spaces. The contrast-dye reached lumbar regions in $42 \%$ of the injections (5/12). A larger spread of the contrast-dye was not observed after the administration of a second dose of the injectate. No signs of epidural, intrapleural/intrapulmonary, intravascular, or intraabdominal spread were observed. The dissection of the two cadavers confirmed the spread of the contrast-dye along the sympathetic trunk and intercostal spaces.

Conclusion: The administration of $0.3 \mathrm{ml} \mathrm{kg}$-1 of the contrast-dye in the CTPV space resulted in a distribution compatible with the block of nerves responsible for the innervation of the majority of the abdominal viscera and cranial abdominal wall.
\end{abstract}

Keywords: Caudal thoracic paravertebral catheter, Dog, Regional anesthesia, Ultrasound guided.

\section{Introduction}

The caudal thoracic paravertebral (CTPV) block is a regional anesthesia technique currently used in human medicine to provide perioperative analgesia in abdominal surgical procedures such as laparoscopic cholecystectomy, ventral hernia repair, open renal surgery, and open major gynecological midline surgery among others (Richardson et al., 1995; Naja et al., 2002; El-Boghdadly et al., 2016).

The boundaries of the TPV space are the internal intercostal membrane (IIM) and costotransverse ligament dorsally, the parietal pleura (PL) ventrolaterally, and the vertebral body, intervertebral disk, and intervertebral foramen medially. Therefore, the injection of local anesthetic (LA) into the TPV space blocks the intercostal nerve as it exits the intervertebral foramen, the rami communicantes, and the sympathetic chain, providing somatic and visceral analgesia (Richardson and Lonnqvist, 1998; Karmakar, 2001).
The abdominal wall in the dog is innervated by dorsal and lateral cutaneous branches of spinal nerves from T12 through L3 (Bailey et al., 1984). The abdominal viscera are innervated by afferent fibers that are projected to the central nervous system) via sympathetic and parasympathetic nerves. In the abdomen, the parasympathetic nerves are involved in regulatory autonomic reflexes, whereas abdominal visceral pain is mediated by the sympathetic nerves. Both caudal thoracic and cranial lumbar dorsal root ganglia reach the majority of the abdominal viscera via the thoracic sympathetic chain and the peripheral plexuses (Cervero, 1994).

In human anesthesia, the use of TPV catheters has been shown to provide effective analgesia for the pain management in the postoperative period (Copic et al., 2017; Liu et al., 2017).

An ultrasound (US)-guided TPV block technique has recently been described in Canidae at the level of the 5th thoracic vertebrae (Monticelli et al., 2017; Portela 
et al., 2017; Ferreira et al., 2018). The previous studies aimed distributions compatible with analgesia for thoracic regions and none of them evidenced a caudal distribution of the injectate within the TPV space; therefore, the nerves providing analgesia for the cranial abdomen were not stained. According to the authors' knowledge, a description of a caudal TPV block and catheter placement has not yet been described in dogs. The aims of this study are to describe an ultrasoundguided technique to place caudal TPV catheters in canine cadavers and evaluate the distribution of a contrast-dye solution administered through the catheter. The study hypotheses were first that catheter placement in the CTPV space can be feasible using a US-guided technique and, second, that the distribution of the contrast-dye at this site will reach the caudal thoracic nerves responsible for analgesia of the cranial abdomen.

\section{Material and Methods}

\section{Ethical approval}

This study was performed in accordance with local RVC Ethical approval URN 2017 1744-3.

\section{Preparation of the dogs}

Eight thawed adult beagle cadavers were used in this study. Cadavers were $9.2 \pm 2.0 \mathrm{~kg}$ body total weight with a body condition score between 5 and 7 out of 9 based on the World Small Animal Veterinary Association Global Nutrition Committee scale.

After positioning the dogs in sternal recumbency, the thoracolumbar region was clipped. All the dogs were intubated with a cuffed endotracheal tube. The cuff was inflated until the complete seal of the trachea was achieved. Manual ventilation was started by one operator using an Ambu bag to allow ultrasonographic visualization of the movement of the PL when performing the US-guided TPV block. Five dogs had bilateral catheter placement. Three dogs had unilateral catheter placement on the contralateral site due to a post-mortem skin suturing near to the study area in one of the hemithorax.

\section{Ultrasound-guided TPV block technique}

All the injections and catheter placement were performed by the same operator (RM) using an $\mathrm{S} 9 \mathrm{v}$ Sonoscape ultrasound machine (Sonoscape, Shenzhen, China) with a $10-5 \mathrm{MHz}$ linear transducer covered by a latex-free ultrasound probe cover (US probe cover, Vygon, UK) with ultrasound gel. A mini set for epidural anesthesia with a Tuohy needle $(1.30 \times 80 \mathrm{~mm}, 18 \mathrm{G})$, catheter $(0.85 \times 0.45 \times 1,000 \mathrm{~mm}, 20 \mathrm{G})$, and filter (Perifix ${ }^{\circledR}$ Filter Set) was used. The distal $2 \mathrm{~cm}$ of the multi-orifice catheter was cut prior to its insertion through the needle. This allowed only one distal orifice for the administration of the solution.

The most caudal intercostal space with visualization of pleural movement was the selected point to insert the needle and later the catheter. The last rib was identified by palpation and the transducer was positioned along its long axis. The rib was visualized as a hyperechoic line with posterior acoustic shadowing. The transducer was then moved cranially as many intercostal spaces as we needed until the movement of the PL from manual ventilation could be visualized. Once the most caudal intercostal space with visualization of pleural movement was identified, the transducer was returned to the rib at its caudal aspect. This rib was followed dorsally until the transverse process (TP) was identified. The ultrasound transducer was gradually rotated anticlockwise until the apex of the TPV space was visualized as a wedge-shaped hypoechoic region bordered by the hyperechoic line of the PL below and the internal IIM above (Fig. 1). The final orientation of the transducer prior to the injection was in an oblique sagittal plane (Fig. 2).

The Tuohy needle connected to a syringe containing the contrast-dye solution was advanced into the TPV

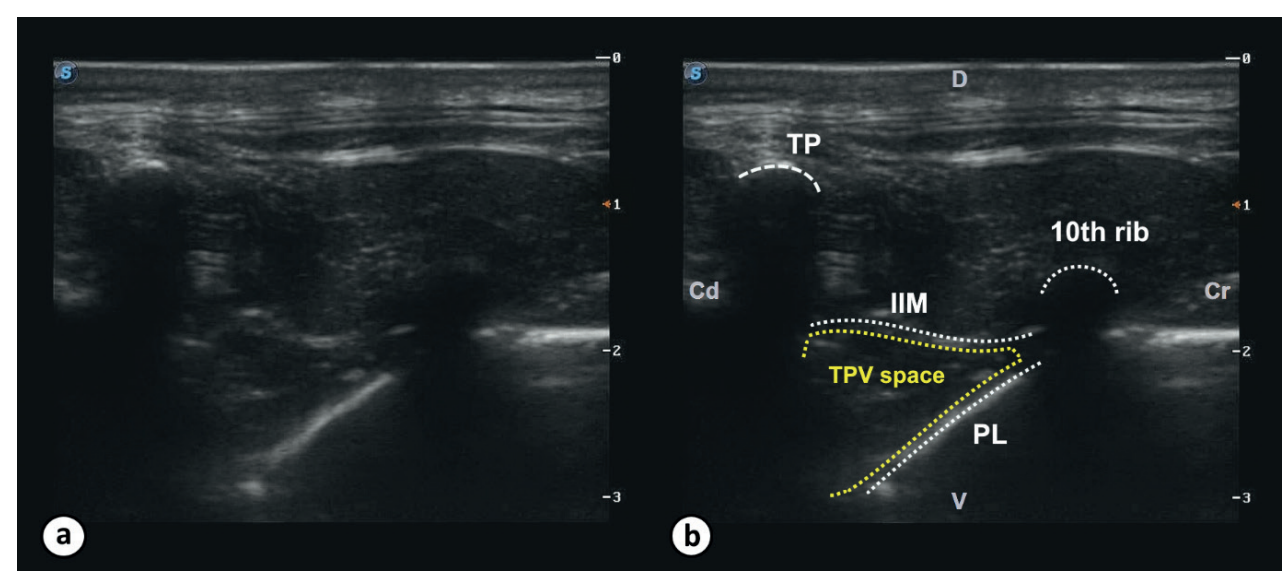

Fig. 1. Ultrasound image of the TPV space in a dog cadaver. The image shows the ultrasonographic appearance (a) with the landmarks for the TPV block (b). The TPV space can be visualized in between the 11th TP and the 10th rib and is delineated by the internal IIM dorsally and the PL ventrolaterally. (Cd): caudal; (Cr): cranial; (D): dorsal; (V): ventral. 

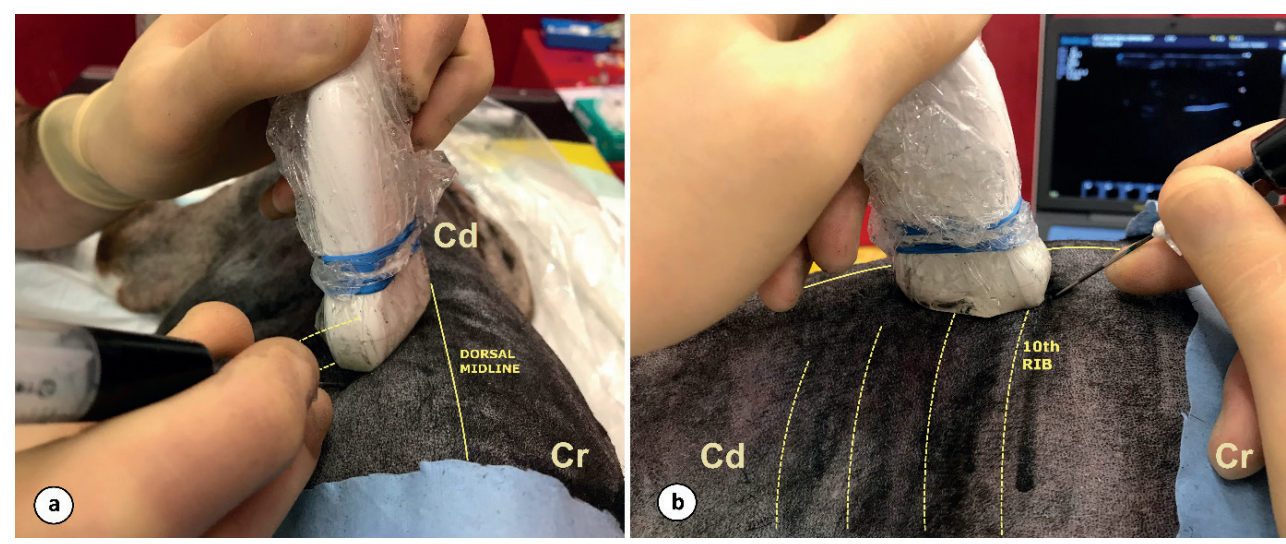

Fig. 2. Final orientation of the transducer prior to the injection of the contrast-dye solution into the TPV space. The transducer is positioned in the oblique sagittal plane to the dorsal midline (a) and the needle is advanced in an in-plane technique in a cranial-to-caudal direction toward the TPV space (b). (Cr): cranial; (Cd): caudal.

space in a cranio-lateral to caudomedial direction from the cranial edge of the rib, at an angle of $45^{\circ}$ to the skin, to puncture the internal IIM using an in-plane technique. The bevel of the needle was orientated away from the PL. Once the tip of the needle was visualized in the TPV space, a 50:50 mixture of $300 \mathrm{mg} / \mathrm{ml}$ iohexol (Omnipaque 300; GE Healthcare, Germany) radiographic contrast and methylene blue (Methylthionium Chloride Injection $1 \% \mathrm{w} / \mathrm{v}$; Martindale Pharmaceuticals Ltd, UK) dye solution was injected.

A total of $0.3 \mathrm{ml} \mathrm{kg}-1$ of the contrast-dye was injected in a first phase (Fig. 3). This volume was divided into two aliquots of $0.15 \mathrm{ml} \mathrm{kg}^{-1}$ injected during the catheter placement as described below. An initial bolus of $0.15 \mathrm{ml} \mathrm{kg}{ }^{-1}$ of the contrast-dye solution was administered through the Tuohy needle after negative aspiration to create a "pocket" (structures within the TPV space were distended after the initial contrastdye injection creating a small pocket of fluid) and ease the posterior advancement of the catheter. Ventral displacement of the pleural membrane was considered as a confirmatory sign of positioning of the tip of the needle within the TPV space. Thereafter, the catheter was introduced through the Tuohy needle and was advanced 1-2 $\mathrm{cm}$ beyond its bevel. In order to advance the catheter through the Tuohy bevel, the angle of the needle was slightly adapted to a less steep angle so that the catheter would be in plane with the TPV space. With the catheter in place within the TPV space, the Tuohy needle was removed and the remaining $0.15 \mathrm{~mL} \mathrm{~kg}^{-1}$ of the contrast-dye was administered. Once a total of $0.3 \mathrm{~mL} \mathrm{~kg}^{-1}$ of the contrast-dye was injected, a computed tomography (CT) scan was carried out to assess the spreading of the contrast-dye.

In a second phase, an extra $0.2 \mathrm{ml} \mathrm{kg}^{-1}$ of the contrastdye was administered through the catheter in five cadavers (eight catheters), immediately following

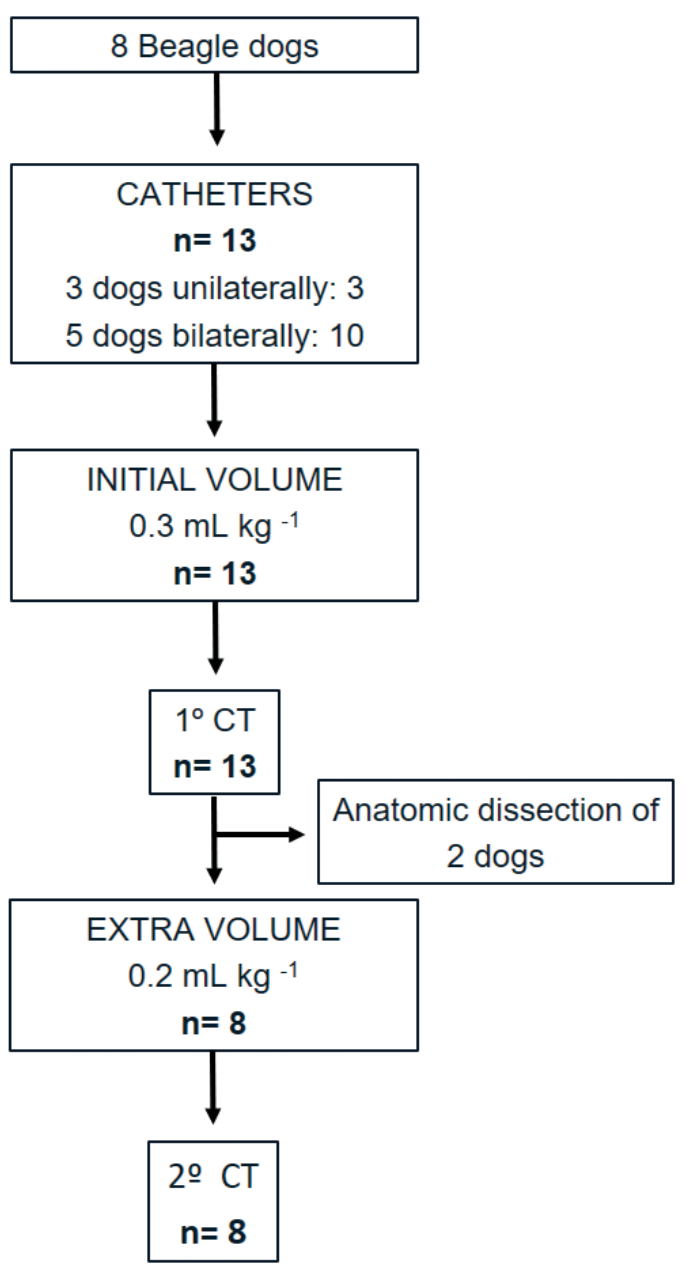

Fig. 3. Flow-diagram representing the number of catheters placed (unilateral and bilateral catheter placement), volumes administered (initial and extra volume), CT study (after initial and extra volume), and anatomic dissection. 
the first CT scan was obtained. Following this extra administration, a second CT scan was carried out in order to assess the spreading of a higher volume of the contrast-dye.

\section{Imaging study and Dissection}

A CT scan of the thorax and abdomen was performed and evaluated by a different operator (MP), maintaining the dogs in sternal recumbency. All scans were obtained using a 16 slice multiple detector CT scanner (MX 8000 IDT; Philips Medical Systems, OH). The CT images were acquired with helical acquisition, slice thickness of $3 \mathrm{~mm}$, image reconstruction interval of $1.5 \mathrm{~mm}$, helical pitch of 0.688 , tube rotation time of 0.75 second, tube current of $150 \mathrm{mAs}$, tube potential of $120 \mathrm{kVp}$, matrix 512512 , and medium frequency ("soft tissue") reconstruction algorithm. Images were reviewed using a bone window (window level 300, window width 1500).

The following CT features were recorded: Paravertebral contrast location (yes/no), distribution of the contrast in relation to the site of injection (number of spinal nerves cranial and caudal), contrast distribution pattern (linear/ intercostal/cloud), mediastinal contrast contamination (yes/no), epidural contrast migration (yes/no), intrapleural/intrapulmonary puncture (yes/no), intravascular injection (yes/no), and intraabdominal injection (yes/no).

Once the CT scans were performed, two cadavers were dissected to assess the distribution of the contrast-dye. The dissection was performed with the dogs in dorsal recumbency. The costochondral junctions were identified by palpation and the overlying skin was reflected. The muscles of the thoracic wall were dissected as appropriate to allow the osteotome to cut the ribs at the costochondral junctions in each hemithorax.

Once all the ribs were cut, a linear incision of the cranial abdomen in between the last costochondral junctions was performed, dissecting the skin, rectus abdominis, external abdominal oblique, internal abdominal oblique, transversus abdominis, and peritoneum. A linear incision between the first costochondral junctions at the thoracic inlet was also performed, allowing the ventral part of the thoracic cage to be removed in order to expose the thoracic cavity. The thoracic viscera were displaced in order to visualize the sympathetic chain and the intercostal nerves through the parietal PL and both endothoracic fascia and parietal PL when appropriate. After the exploration of the stain, the parietal PL and endothoracic fascia were dissected to allow direct visualization of the catheter within the TPV space and the stain of the sympathetic trunk and intercostal nerves.

\section{Statistical analysis}

Data were analyzed using IBM SPSS Statistic for Windows 21.0 (IBM Corp., NY). Normality was assessed using the Shapiro-Wilk test. Descriptive statistics were used. Mean and standard deviation are reported for parametric data. Median and range are reported for non-parametric data. Differences were considered statistically significant with $p<0.05$.

\section{Results}

The injection site varied between T8-9 and T12-13. Contrast was identified in the paravertebral space in $92 \%$ of the injections (12/13). The right injection in dog number 7 was directly performed in the mediastinum. The distribution pattern observed in all the injections within the TPV space (12/12) was linear and intercostal (Fig. 4). The median (range) linear spread of the contrast was 7 (5-10) spinal nerves and involved $3(2-8)$ intercostal spaces. Forty-two percent of the injections within the TPV space (5/12) were distributed beyond the psoas muscle origin, reaching lumbar regions. The sites of injection and the spread of the contrast-dye within the TPV space are illustrated in Figure 5.

A total of nine catheters out of ten could be advanced beyond the tip of the Tuohy needle. In 78\% (7/9) of these cases, injection resulted in mediastinal contamination. The linear and intercostal spread of contrast was not extended in any of the eight injections in which extra volume $\left(0.2 \mathrm{~mL} \mathrm{k}^{-1}\right)$ was administered. The contralateral spread was observed from the right catheter in dog number 5 after the extra volume was injected.

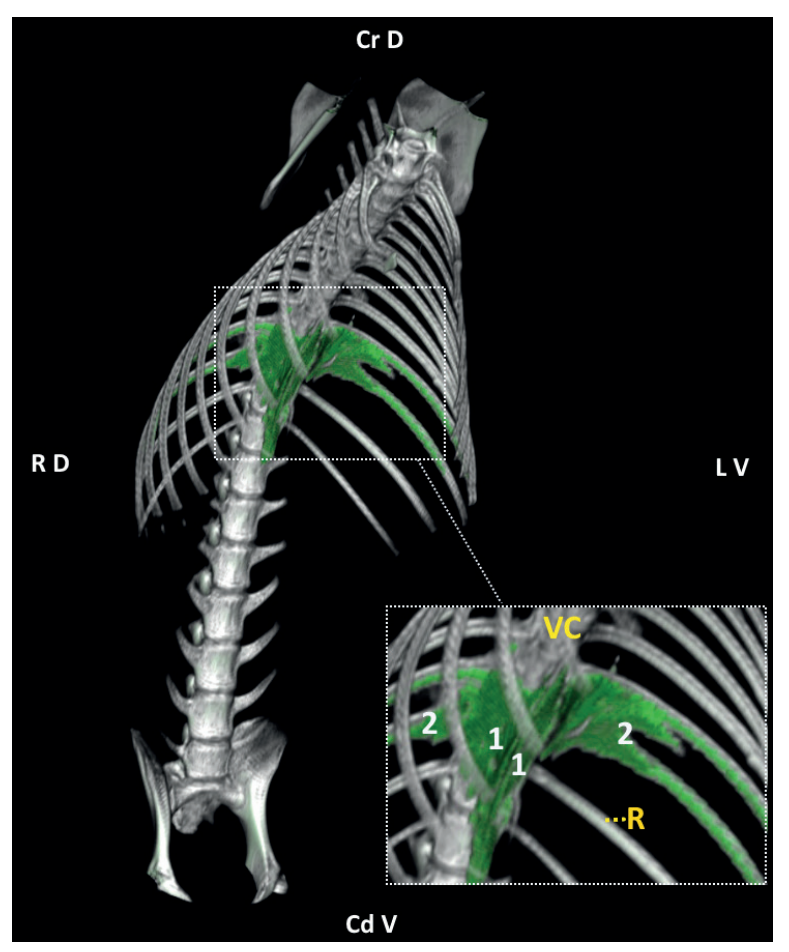

Fig. 4. Three-dimensional volume rendering of the CT imaging study showing the linear (1) and intercostal (2) distribution of the contrast (green appearance) for the dog number 8 (injection site T12-T13). (R): 12th rib; (VC): vertebral column. See orientation landmarks as follows: $(\mathrm{Cd}$ V): caudo-ventral; (Cr D): cranio-dorsal; (LV): left-ventral; (RD): right-dorsal. 
No signs of epidural, intrapleural/intrapulmonary, intravascular, or intraabdominal contrast spread were observed in this study.

The dissection of the two cadavers after the administration of the contrast-dye confirmed the position of the catheters inside the TPV space as well as the multisegmental spread of contrast-dye along the sympathetic trunk and intercostal spaces (Fig. 6).

\section{Discussion}

In spite of the small number of dogs used in this study, our results showed that the described technique could be a suitable method to place TPV catheters in the caudal area of the thorax. Although the placement of catheters in the TPV space has been described as challenging by some authors in the human literature (Luyet et al., 2009), its use has considerably increased in recent years. Clear sonographic images of the TPV space and the tip of the needle were obtained during the placement of all catheters. The ventral displacement of the PL was visualized during all the ultrasound-guided injections and was regarded as a confirmatory sign of accurate needle placement within the TPV space, as described previously by other authors (Monticelli et al., 2017;

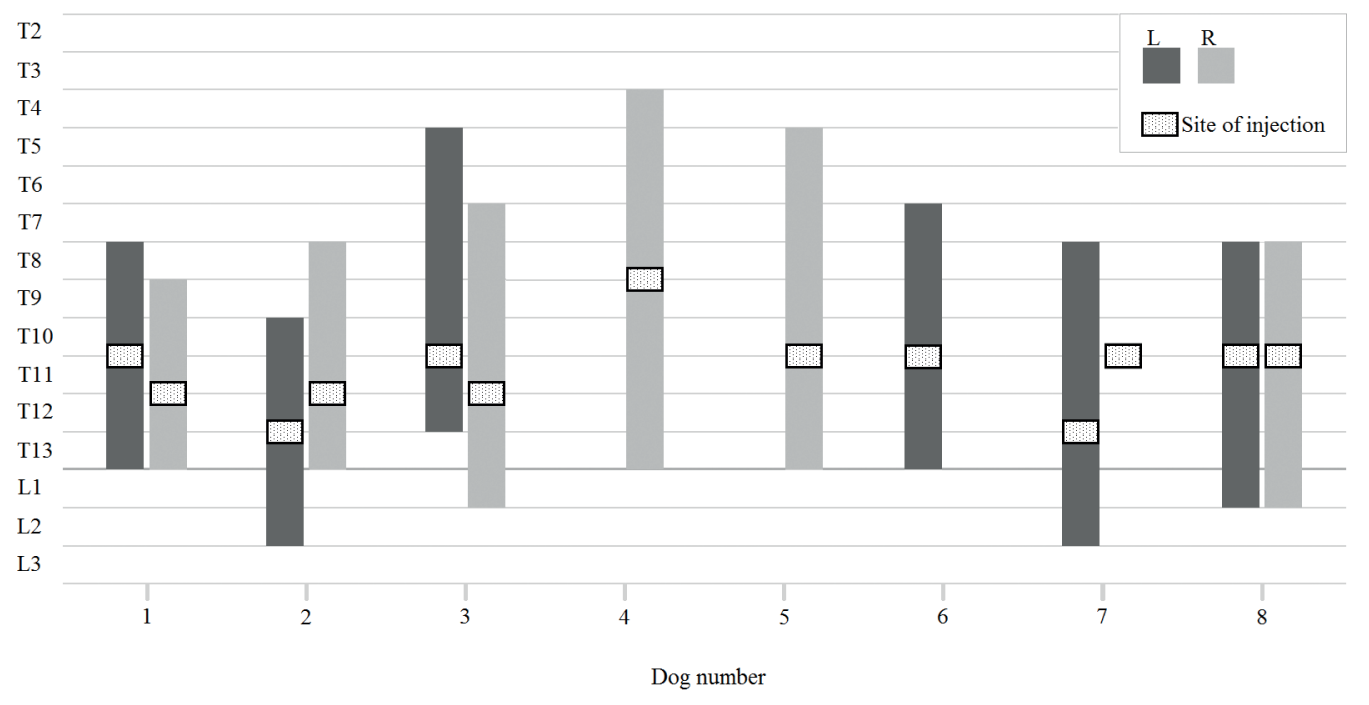

Fig. 5. Schematic diagram showing the site of injection (dotted square) and the spread of contrast within the left (L) and right (R) TPV space for each dog. The R injection in dog number 7 was not found within the TPV space.
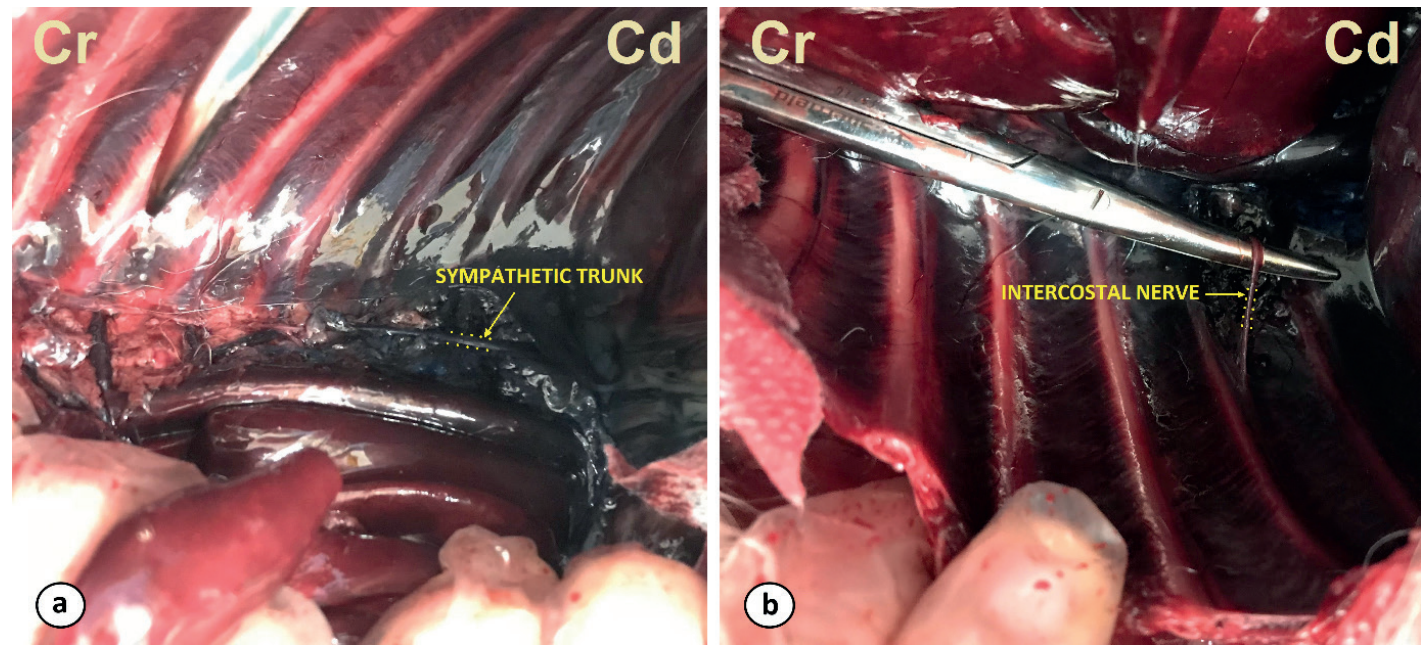

Fig. 6. Ventral views of the cadavers after the dissection and removal of the ventral part of the thoracic cage. The dye of the sympathetic trunk (a) and the intercostal nerve (b) are shown. (Cd): caudal; (Cr): cranial. 
Portela et al., 2017; Ferreira et al., 2018). Three dogs had post-mortem suturing of the skin near to the study area in one of the hemithorax. The air entrapped in the subcutaneous tissue on the affected site of these dogs was the origin of reverberations of the ultrasound waves, hindering identification of the relevant structures subject of this study. It was decided not to use the affected sites in these three dogs, therefore having unilateral catheter placement at the unaffected site.

One limitation of this study was the lack of standardization of the injection site. The injections varied between T8-9 and T12-13. As the ribs become more caudally orientated in the caudal thoracic region, the ultrasonographic window between the TP and the adjacent rib is narrowed. Therefore, the orientation of the injection in this study was cranio-lateral to caudomedial. Visualization of the PL was crucial to insert the needle within the TPVS. In some cadavers, the pleural movements (gliding sign) generated by manual ventilation were not easily identified in the caudal intercostal spaces. In some dogs, the movement of the PL was not identified during manual ventilation in the caudal intercostal spaces. In these cases, cranial intercostal spaces with clear sonographic images were selected for the injection and catheter placement. Postmortem tissue changes could have led to abnormalities in the pleural movement and distension of the lungs. Also, the manual ventilation might not have been efficient enough to re-expand the atelectatic lungs. Lung recruitment strategies or the instauration of mechanical ventilation could have improved the visualization of the gliding sign in the US images and help to identify the PL in the caudal areas. For these reasons, and to ensure clear sonographic images at the injection site, the authors' selected different injection sites in this study. In a recent review of the use of the TPV block in abdominal procedures in humans, the site of injection among the studies varied from T5 to T12 when performing the blocks in the thoracic region, depending on the intended area of analgesia (ElBoghdadly et al., 2016).

In the present study, $0.3 \mathrm{ml} \mathrm{kg}^{-1}$ resulted in a linear distribution of 7 (5-10) spinal nerves. This distribution is greater than that reported in previous studies where different volumes were used. The linear spread of the injected solution in these studies varied from a mean of $5.0 \pm 1.5$ intercostal segments (Monticelli et al., 2017) when $0.2 \mathrm{ml} \mathrm{kg}^{-1}$ was used; a median (range) of $3(0-3)$ to 3.5 (1-6) vertebral bodies (Portela et al., 2017) when 0.05 and $0.15 \mathrm{ml} \mathrm{kg}{ }^{-1}$ were used, respectively; and a median (range) of $0(0-2)$ and $1.5(0-4)$ vertebral spinal levels (Ferreira et al., 2018) when 0.1 and $0.3 \mathrm{ml} \mathrm{kg}^{-1}$ were used, respectively. The administration of an extra volume of $0.2 \mathrm{ml} \mathrm{kg}^{-1}$ in the current study did not result in a more extended longitudinal distribution of the contrast-dye.

Furthermore, the median (range) spreading of the contrast-dye along the intercostal spaces observed in our study was of $3(2-8)$ intercostal spaces. This distribution was larger than the reported in other studies where either only the spinal nerve at the injection site was stained (Portela et al., 2017), or only a median (range) of $1(1-2)$ and 1 (1-3) intercostal spaces (after the injection of 0.1 and $0.3 \mathrm{ml} \mathrm{kg} \mathrm{kg}^{-1}$, respectively) contained the injected solution (Ferreira et al., 2018). The authors in the aforementioned studies stipulated that the limited multisegmental and intercostal spread could be the result of the injection of the solutions either in the extrapleural paravertebral or subendothoracic paravertebral compartments. Also, another explanation is that the injections may have been performed more laterally toward the intercostal space, thus limiting the spread of dye medially (Ferreira et al., 2018).

Recently, the boundaries of the TPV space have been studied in human medicine. These boundaries were the internal IIM and superior costotransverse ligament on the posterior side, the parietal PL and the endothoracic fascia in the anterolateral side and the vertebral body, intervertebral disk, and foramen in the medial side (Bouman et al., 2017). The pattern of distribution observed in our study was consistent with these findings, where the separation of the TPV space in a subendothoracic and extrapleural compartment was not observed and the TPV space had a lateral communication with the intercostal space. None of the previous studies observed the lumbar spread of the injected solution (Monticelli et al., 2017; Portela et al., 2017; Ferreira et al., 2018). Despite this, there are previous reports attributing the origin of the psoas muscle as the caudal boundary of the TPV space in humans (Lönnqvist and Hildingsson, 1992), and recently Bouman et al. (2017) showed a spread of dye into the lumbar paravertebral space. In our study, $42 \%$ of the injections within the TPV space $(5 / 12)$ were distributed beyond the psoas muscle origin, reaching lumbar regions. The communication of the TPV space with the lumbar paravertebral space has been previously reported in a human study (Bouman et al., 2017). The communication between these two spaces was observed posteriorly into the costodiaphragmatic recess and could be explained by the fact that the endothoracic fascia was continued as the transversalis fascia on the abdominal region. The distribution showed in the present study may be compatible with the block of areas responsible for the innervation of the majority of the abdominal viscera and the cranial abdominal wall in dogs. Bilateral catheter placement could then provide analgesia for non-surgical conditions as pancreatitis and urinary obstruction, or surgical conditions as nephrectomies, cystectomies, and enterectomies among others.

We opted to advance the catheters as a part of our standard practice to prevent catheter dislocation. In this study, the further advancement of the catheters through the tip of the Tuohy needle offered resistance and was considered challenging, in contrast to the easy advancement of the needle into the TPV space. 
None of the catheters left at the tip of the Tuohy needle (4/13) resulted in mediastinal contrast contamination, whereas the $78 \%$ of the catheters advanced beyond the Tuohy bevel (7/9) led to mediastinal contrast contamination. Previous studies on the TPV block in canidae report mediastinal contamination in $42 \%$ of the injections (Monticelli et al., 2017). The mediastinum consists of two serous membranes that extend cranially from the thoracic inlet and caudally to the diaphragm, being attached dorsally to the thoracic vertebrae and ventrally to the sternum (Nikel et al., 1979). In some anatomy reference texts, the sympathetic trunk may be considered to be located within the mediastinum (Nikel et al., 1979), whereas other texts do not consider this structure to be within the mediastinum (Evans and de Lahunta, 2012). Therefore, the significance of mediastinal contamination is uncertain given that some structures of the TPV space may be considered within the mediastinum.

A technical limitation of the technique for catheter placement is the potential displacement of the catheter when removing the Tuohy needle. The authors decided to divide the total volume used during the catheter placement $\left(3 \mathrm{ml} \mathrm{kg}^{-1}\right)$ in two aliquots of $0.15 \mathrm{ml} \mathrm{kg}^{-1}$ in order to create a "pocket" or distend the structures within the TPV with the contrast-dye to ease the placement of the catheter. This could have influenced the distribution of the contrast-dye.

As another limitation of our study, a complete comparison of the distribution between the imaging study and the dissection of the cadavers was not achievable as only two cadavers were dissected. Although contralateral migration of contrast is deemed unlikely (Bouman et al., 2017) and has not been documented in the previous ultrasoundguided TPV studies in veterinary medicine (Monticelli et al., 2017; Portela et al., 2017; Ferreira et al., 2018), we found contralateral spread after the administration of the extra volume in one dog. The authors speculate that this could be the result of direct iatrogenic injection from the contralateral TPV space. Another limitation for our study was performing the CT exam after injection of the contrast-dye on both sides. This methodology could have interfered with/complicated the interpretation of the CT scans in the dogs with bilateral catheter implantation due to the possible crossover of contrast-dye to the other side. In conclusion, the placement of a TPV catheter was considered successful in the present cadaveric study in dogs. When used bilaterally, the administration of 0.3 $\mathrm{ml} \mathrm{kg}{ }^{-1}$ of the contrast-dye solution in the CTPV space resulted in a distribution compatible with the block of areas responsible for the innervation of the majority of the abdominal viscera and cranial abdominal wall. In addition, the authors would suggest positioning the tip of the catheter at the same level as the tip of the Tuohy needle. Future studies will be required to evaluate its use in a clinical scenario.

Conflict of interest

Authors declare no conflict of interest.

\section{Authors' contributions}

RMS, PM, and JV: Conceived and participated in the design, execution of the study, and data collection. MP participated in the execution of the study and data collection. RMS, MP, CPJ, and JV contributed to the writing of the manuscript.

\section{References}

Bailey, C.S., Kitchell, R.L., Haghighi, S.S. and Johnson, R.D. 1984. Cutaneous innervation of the thorax and abdomen of the dog. Am. J. Vet. Res. 45, 1689-1698.

Bouman, E.A.C., Sieben, J.M., Balthasar, A.J.R., Joosten, E.A., Gramke, H.F., Kleef, M.V. and Lataster, A. 2017. Boundaries of the thoracic paravertebral space: potential risks and benefits of the thoracic paravertebral block from an anatomical perspective. Surg. Radiol. Anat. 39, 1117-1125.

Cervero, F., 1994. Sensory innervation of the viscera: peripheral basis of visceral pain. Physiol. Rev. 74, 95-138.

Copic, M., Bialka, S., Daszkiewicz, A. and Misiolek, H. 2017. Thoracic paravertebral block for postoperative pain management after renal surgery. Eur. J. Anaesthetsiol. 34, 596-601.

El-Boghdadly, K., Madjdpour, C. and Chin, K. 2016. Thoracic paravertebral blocks in abdominal surgery - a systematic review of randomized controlled trials. Br. J. Anaesth. 117, 297-308.

Evans, H.E. and de Lahunta, A. 2012. Miller's Anatomy of the dog, 4th ed. Saunders, UK, pp: 352-355.

Ferreira, T.H., Teixeira, L.B.C., Schroeder, C.A., Garcia C.deM. and Schroeder, K.M. 2018. Description of an ultrasound-guided thoracic paravertebral block technique and the spread of dye in dog cadavers. Vet. Anaesth. Analg. 45, 811-819.

Karmakar, M. K. 2001. Thoracic Paravertebral Block. Anesthesiology 95, 771-780.

Liu, F., Zhang, H., and Zuo, Y. 2017. Bilateral thoracic Paravertebral block for immediate postoperative pain relief in the PACU: a prospective, observational study. BMC Anesthesiol. 17, 89.

Lönnqvist, P.A. and Hildingsson, U. 1992. The caudal boundary of the thoracic paravertebral space. A study in human cadavers. Anaesthesia 47, 1051-1052.

Luyet, C., Eichenberger, U., Greif, R., Voqt, A. and Szücs, F.Z. 2009. Ultrasound-guided paravertebral puncture and placement of catheters in human cadavers: an imaging study. Br. J. Anaesth. 102, 534-539.

Monticelli, P., Jones, I. and Viscasillas, J. 2017. Ultrasound-guided thoracic paravertebral block: cadaveric study in foxes (Vulpes vulpes). Vet. Anaesth. Analg. 44, 968-972.

Naja, Z., Ziade, M.F. and Lonnqvist, P.A. 2002. Bilateral paravertebral somatic nerve block for ventral hernia repair. Eur. J. Anaesthetsiol. 19, 197-202. 
Nikel, R., Schummer, A. and Seiferla, E. 1979. The Viscera of the Domestic Mammals. Singhofen, Germany: Verlag Paul Parey, pp: 2-20.

Portela, D.A., Campoy, L., Otero, P.E., Martin-Flores, M. and Gleed, R.D. 2017. Ultrasound-guided thoracic paravertebral injection in dogs: a cadaveric study. Vet. Anaesth. Analg. 44, 636-645.
Richardson, J., Vowden, P. and Sabanathan, S. 1995. Bilateral paravertebral analgesia for major abdominal surgery: a preliminary report. Anaesthesia 50, 995-998.

Richardson, J. and Lonnqvist, P.A. 1998. Thoracic paravertebral block. Br. J. Anaesth. 81, 230-238. 